\title{
Teaching and Learning of Algebra
}

\author{
Rakhi Banerjee and Luis Puig
}

\section{Overview}

Topic Study Group 9 aimed to bring together researchers, developers and teachers who investigate and develop theoretical accounts of the teaching and learning of algebra. The group sought both empirically grounded contributions focussing on the learning and teaching of algebra in diverse classrooms settings, the evolution of algebraic reasoning from elementary through university schooling as well as theoretical contributions throwing light on the complexities involved in teaching and learning of algebra. Prospective contributors were requested to address one or more of the following themes: early algebra, use of ICT in algebra classrooms, proof and proving in algebra, problem solving, semiotics, designing of algebra curriculum.

\section{Organization}

We received 44 contributions for the TSG. Based on the review of these papers (each paper was reviewed by two members of the organizing team), 25 of these contributions were chosen for oral presentations and the rest were recommended for

Co-chairs: Rakhi Banerjee (India) rakhi.banerjee@gmail.com and Luis Puig (Spain) luis. puig@uv.es

Team Members: Swee Fong Ng (Singapore) sweefong.ng@nie.edu.sg, Armando Solares (Mexico) asolares@cinvestav.mx, Hwakyung Kim (Korea) hwakyung@gmail.com, Maria Blanton (USA)

R. Banerjee ( $\square)$

Azim Premji University, Bangalore, India

e-mail: rakhi.banerjee@gmail.com

L. Puig

Universitat de València Estudi General, Valencia, Spain

e-mail: luis.puig@uv.es

(C) The Author(s) 2015 
poster presentations. 16 of the oral presentations were short presentations $(10 \mathrm{~min}$ for presentation and $5 \mathrm{~min}$ for discussion) and 9 were long presentations (20 $\mathrm{min}$ for presentation and $10 \mathrm{~min}$ discussion). For reasons of optimizing the available time and in order to fit in all the contributions, the group was divided into two subgroups and presentations were made simultaneously in the two sub-groups. The co-chairs of the team often helped in identifying the underlying theme in different presentations across the two sub-groups. Participants were requested to read up the articles to be presented in a session beforehand to be able to participate better. Some time was kept daily for the whole group to meet and discuss issues arising from the presentations or points which participants wanted to raise. More time was allotted for whole group activity on the first and the last day.

The participants were largely those who had contributed to the group and brought in perspectives from all over the world. The presentations touched upon students' understanding of different aspects of algebra, theoretical perspectives to make sense of students' work and help them learn better, teachers' understanding of the algebra they teach and professional development initiatives to help them focus on the important aspects of algebra. Pattern generalization and early algebraic thinking was an issue of discussion in various presentations. Problem solving and reasoning, proving, understanding of functions were explored in a few presentations. There were a couple of reports on algebra in particular culture/communities and curriculum/instruction status in a country. A few presentations focused on the use of computer aided tools for instruction or evaluation. An area which did not get any attention was how semiotics helps us understand students' developing knowledge of symbols, process of signification and communication.

\section{Implementation}

\section{Session 1: July 10, Tuesday, 10:30-12:00 (Room no. 308a and 309)}

On the first day, 45 min were kept for whole group discussion and only 4 presentations were scheduled for oral communication (2 long presentations and 2 short ones). The participants were reminded of the themes that the TSG would focus on and were given a general overview of the nature of the submissions received. They were further informed about the modalities of the conduct of the TSG.

The four presentations covered varied themes. One of the presentations focused on understanding of properties of operations with respect to fractions, operations on fractions, ability to think relationally and to perceive structure in expressions and their importance for learning algebra and developing algebraic thinking. Another one looked at the usefulness of variation theory as a means of improving teaching and learning and discussed how teachers went about designing lessons in the area of 
rational expressions using the theory. A third presentation looked at pre-service teachers' ability to engage in inductive reasoning and generalization in problem solving contexts. The fourth presentation focused on professional development of teachers in the area of functions that helped them develop and design activities that promote algebraic thinking among students.

Thus, we listened to an interesting set of ideas in terms of design of tasks, theoretical frameworks on the first day. They highlighted strengths and limitations of teachers' and students' thinking and reasoning while working on the tasks and provide valuable insights for designing of programmes with teachers and students in the future.

\section{Session 2: July 11, Wednesday, 10:30-12:00, Room no. 308a and 309}

Eight presentations were scheduled for the second day, four of them were long presentations and four short ones, divided equally between the two rooms.

All the presentations in one of the rooms dealt with pattern generalization. One of them discussed strategies used by students in secondary school for generalizing two patterns. Another one looked at difference in performance among students categorized by their abilities in mathematics on pattern generalization tasks as well as the strategies used for working on the tasks. One study compared competence of students in two countries: Hongkong and United States, in pattern generalization task. A last paper explored young Australian indigenous students' engagement with generalization of contextual growing patterns and ways in which cultural gestures help them in accomplishing the task.

The studies highlighted many categories of patterns and strategies for generalizing them and the participants discussed issues arising out of pattern generalizing tasks in their own countries and classrooms and made suggestions towards improving students' abilities to generalize, nature of tasks and instructions for students etc.

Presentations in the second room were not in a single topic. The long one dealt with algebraic proof in secondary education. In this presentation findings of a teaching experiment were reported on how the understanding of the generality of algebraic proof emerged when students study operative proofs. The study started from the knowledge from previous research that even students who are able to construct proofs using symbolic algebra rely on checking with numerical examples as a "proof". Students that followed the experimental teaching, that included the use of operative proofs, start noticing the generality of operation and then they start appreciating algebraic proofs.

The short ones dealt with very different issues. One presented a proposal to describe the structure of algebraic competence by using linear structure models. The second one dealt with algebraic reasoning in early algebra as generalized arithmetic, 
examining elementary school students' understanding of the properties of whole numbers operations. The reflection of students on the properties of operations with whole numbers is a way to teach and learn algebraic reasoning in early ages. In the study, it was found that students had capabilities in generalizing the properties of numbers and operations, but they had not developed such capabilities, because school practices have not provided enough opportunities and experience in order to develop them. This was showed by the fact that student were able to generalize the commutative law, but neither the associative nor the distributive laws.

The final one dealt with a research study on the ability of secondary students to translate statements between symbolic algebra and vernacular language and viceversa. In this study, students performed better when translating from symbolic algebra expressions to vernacular language, and most errors when translating the other way round were attributed by the authors to "peculiar features of algebraic language".

\section{Session 3: July 13, Friday, 15:00-16:30, Room no. 308a and 309}

Eight more presentations were scheduled for this day, in a manner similar to Session 2.

Two presentations in one of the rooms highlighted students' capacities to reason algebraically in various situations. One of the presentations shared a teaching experiment aimed to promote the development of algebraic thinking among grade 4 students in the context of identifying numerical relations and patterns and thus deriving generalizations. Another one talked about an online game which focused on developing students' (grade 6) abilities to solve contextual problems dealing with covariation and functional relations and thus enter the domain of algebraic thinking. The other two focused on curricular issues. The third presentation analysed the differences in the treatment of the concept of function in two different kinds of middle school curricula used in the United States. The last presentation dealt with the status of algebra instruction, and in particular instruction of equations, in China where the author examined the textbooks, students' learning and teachers' instruction to come to understand the issue.

The four presentations in the other room focused on varied themes in algebra education. The first presentation briefed on a part of a larger study trying to understand the relationship between students' understanding of fractions as quantities and their abilities to form equations which require such multiplicative understanding. The second presentation reported on students' understanding of function concept among nursing students after they had worked in a context-based, collaborative instructional module. The third reported freshmen students' ability to use their algebra pre-requisite skills while working on calculus problems (Applied calculus optimization problem). The last presentation explored elementary school 
students' non-formal algebraic reasoning while solving word problems, especially by focusing on the mathematical structure or attending to the relation between quantities in the problem.

\section{Session 4: July 14, Saturday, 10:30-12:00, Room no. 308a and 309}

We had scheduled five presentations on the last day, however one of the presenters did not show up, so we ended with four presentations, two long and two short. The first paper in one of the rooms presented a theoretical framework to account for the difference in performance of students who have been taught problem solving through a particular heuristic of drawing a diagram and its impact on their ability to use letter-symbols later in learning algebra. The other presentation highlighted the use of geometrical method in a dynamic environment while solving quadratic equations.

The short presentation in the other room analyzed secondary school students' structure sense, while they had to reproduce rational expressions involving identities. The long one addressed the use of ICT for diagnostic and differentiation purposes, by presenting an online set of resources to diagnose students' knowledge on algebra, and to provide teachers with appropriate resources for managing a differentiated algebra curriculum to meet students' different needs.

A wide range of issues thus got addressed through the presentations and led to fruitful and engaging discussions. These highlighted the abilities and limitations of children's/students' understanding in various conditions; teachers' understanding and role in developing algebraic thinking; the effects of curriculum, textbooks, tasks and technology in promoting students' understanding and teachers' abilities to teach effectively. Participants often related their own experiences within their countries. An interesting pattern that emerged from the presentations and discussions was the way Asian countries emphasise and inculcate the use of symbols and symbolic writing from an early age, whereas this is a much delayed activity in US and many parts of Europe. Thus, the research studies also looked for evidences of having achieved this competence and/or ways to strengthen it. The western countries look for emerging symbols and idiosyncratic use of symbols among children to elucidate their reasoning and thinking processes. This eventually leads them to develop a better understanding of symbols and systematic use of them at a later stage. 


\section{Conclusion}

We did of course face some difficulties in organizing the TSG. The organizing team worked quite well before the conference in giving inputs and reviewing the proposal submissions in time. However, the actual organization was not very simple. The breaking into subgroups although helped us logistically, we lost on listening to each speaker and had to satisfy with the summaries presented by them during the whole group discussion. This would have been more fruitful had everyone read the papers before the session, which was rarely the case. Unfortunately, often due to limited capacities of participants to express in the English language, summaries or discussions could not be taken beyond a point and those who had facility with this language were the ones who got heard more. Some more time or some other ways of organizing the TSG may prove to be more fruitful. Since all the presentations in every TSG were scheduled well in advance and all participants knew the exact listing of presentations by speakers, participants moved from one to another TSG. Thus, the group kept changing each day making it difficult to engage in themes, issues and concerns of a particular TSG.

Open Access This chapter is distributed under the terms of the Creative Commons Attribution Noncommercial License, which permits any noncommercial use, distribution, and reproduction in any medium, provided the original author(s) and source are credited. 